\title{
Influence of Affordances in the Home Environment on Motor Development of Young Children in Japan
}

\author{
Shiro Mori, ${ }^{1}$ Hiroki Nakamoto, ${ }^{1}$ Hiroshi Mizuochi, ${ }^{2}$ Sachi Ikudome, ${ }^{1}$ and Carl Gabbard ${ }^{3}$ \\ ${ }^{1}$ National Institute of Fitness and Sports in Kanoya, Faculty of Physical Education, 1 Shiromizu-cho, Kanoya, Kagoshima, Japan \\ ${ }^{2}$ Child Care St. Mary's College, Nagoya, 2-54 Myogetsu-cho, Shouwa-ku, Nagoya, Aichi, Japan \\ ${ }^{3}$ Texas A\&M University, Department of Health \& Kinesiology, College Station, TX 77843, USA
}

Correspondence should be addressed to Carl Gabbard; c-gabbard@tamu.edu

Received 14 May 2013; Accepted 22 July 2013

Academic Editor: Annie Vinter

Copyright (C) 2013 Shiro Mori et al. This is an open access article distributed under the Creative Commons Attribution License, which permits unrestricted use, distribution, and reproduction in any medium, provided the original work is properly cited.

\begin{abstract}
Previous research indicates that the home environment is a significant factor in early child development. The present study examined influence of the multidimensional home environment on young Japanese children's motor development. A Japanese translation of the Affordances in the Home Environment for Motor Development-Self Report (AHEMD-SR) was used to assess home motor affordances in 262 families. Motor ability was assessed by parental report using the Enjoji Infant Analytic Developmental Test. We also asked parents to rate their own physical activity in terms of level and years of experience. As results, we found that the home environment in Japan was generally sufficient for children's motor development and that children's access to Fine Motor Toys (FMT) and Gross Motor Toys (GMT) had the strongest influence on their development. Analysis also indicated that AHEMD-SR scores were higher for children of parents who had some level of physical activity experience compared to children whose parents indicated no physical activity experience. Parents' self-reported activity level was correlated with higher scores for the subscales FMT and GMT and for total AHEMD-SR score. These results indicate that both the physical and social-psychological environments (parental experience and views) of the home influenced children's motor development.
\end{abstract}

\section{Introduction}

In the field of child development, environmental influences are considered critical factors for optimal growth and development. The home environment, in particular, is a primary agent for learning and development in children (see review by Son and Morrison [1]). Interestingly, one of the major findings in studies that assessed the general characteristics of the home and its relationship to a child's later behavior is that toy availability was a strong predictor of future mental behavior $[2,3]$. Subsequent studies have focused on toy availability and motor development [2-4]; however, little is known about the multidimensional home environment and motor development. The multidimensional home environment includes not only physical factors (such as movement play equipment in the home and the presence of a nearby playground), but also social-psychological factors (such as number of playmates, family composition, and amount of parental attention). In addition, affordance is a fundamental concept in the ecological (affordance) theory for action [5, 6]. Hirose [7] stated that "affordances are opportunities for action that objects, events, or places in the environment provide for the animal." That is, action can be stimulated by the environment, and home environments provide opportunities (affordances) for action, thereby stimulating motor development.

Rodrigues and his collaborators [8] developed a parental self-report inventory-the Affordances in the Home Environment for Motor Development-Self Report (AHEMDSR)-based on the concept of affordances. One of the purposes of its creation was to determine the relationship between the multidimensional home environment and motor development $[9,10]$. Data provided by the inventory has aided in clarifying the role of the multidimensional home environment on early childhood motor development and has been used to evaluate and guide the design of home and child-care environments in ways most beneficial for young children's motor development. Translated (from the original 
English) versions of the AHEMD-SR have been used in several countries, including Portugal, Brazil, China, France, The Netherlands, and Lebanon.

Justification for the present study is the fact that minimal research exists in Japan examining the relation between motor development and the home environment during early childhood. One of the few studies to have done so is that of Sugihara and his collaborators [11] which investigated the relationship between motor ability and home environment of children of 4 years or older, reporting that the socialpsychological environment and the physical environment are both related to motor development. Children in homes with more play equipment showed higher levels of motor ability, whereas housing style (e.g., single-family dwelling, apartment) had little relation to motor ability. In regard to social-psychological factors, the study found that children whose parents want them to play professional sports had higher levels of motor ability than children whose parents have no such desire. Furthermore, children whose homeroom teacher was aware of the benefits of physical activity had better motor abilities than children in other classes. These results suggest that parental and caregiver's experiences with regard to physical activity may influence motor development. However, as noted earlier, that study did not examine children younger than 3 years of age nor did it use a validated home motor affordance assessment instrument.

To fill this gap, the present study created a Japanese version of the AHEMD-SR and examined its relationship with young children's motor development and parental experience and views of physical activity.

\section{Methods}

2.1. Participants and Procedures. Questionnaires were mailed directly to 15 facilities (13 nursery schools, 1 kindergarten, and one child-care center) in three Japanese prefectures (Kagoshima, Tokyo, and Hokkaido) where the investigation had been approved. Families with children aged 1842 months whom agreed to participate in the study were given a packet containing the survey items (described below) and instructions for completion. They were asked to return the items within approximately 2 weeks. The final sample consisted of 261 families, with 44 children (16.9\%) in the 1823-month age group, 141 children (54\%) in the 24-35-month age group, and 76 children $(29.1 \%)$ in the 36-42-month age group.

\subsection{Survey Items}

2.2.1. Affordances in the Home Environment for Motor Development-Self Report (AHEMD-SR). The AHEMD-SR (Rodrigues et al. [8]) is a validated and reliable questionnaire that assesses the quality and quantity of factors (opportunities) in the homes that are conducive to enhancing child motor development. Recent reports of its use include [1215]. This inventory consists of 67 items representing 20 variables (19 plus family characteristics) categorized into 5 subscales: Outside Space (OS), Inside Space (IS), variety of stimulation (VS), Fine Motor Toys (FMT), and Gross Motor
Toys (GMT). Within each of those subscales are the following 19 variables: outside surfaces, outside apparatus, inside space, inside apparatus, inside play space, play stimulation, freedom of movements, encouragement of stimulation, daily activities, replica toys, educational toys, games, construction toys, real materials, musical materials, manipulative materials, locomotor materials, body exploration materials, and others.

Three types of questions are used: simple dichotomic choice questions, 4-point Likert-type scales, and descriptionbased queries. The AHEMD-SR was translated from English into Japanese by two researchers in a related field of motor development. The translated contents were examined closely by two researchers and one teacher at a childcare site. A complete view of the instrument can be seen via the AHEMD website <http://www.ese.ipvc.pt/dmh/AHEMD/ ahemd.htm $>$.

\subsubsection{Parents' Physical Experience and Activity Level. Parents} were asked to rank their approximate years of physical activity (PA) experience on a 5-point scale ranging from "none" to " 3 years or more." PA experiences were defined here as those activities engaged in specifically to increase the individual's level of physical health, such as exercising or playing sports. Parents also rated their activity level on a 5-point scale ranging from "very good" to "very weak" (Table 1).

2.2.3. Assessment of Motor Ability. We used 11 items from the locomotor movements section of the Enjoji Infant Analytic Developmental Test, Revised Edition [16, 17]. The instrument is a relatively simple, easily administered test for children from newborn to 7 years of age. The instrument provides six assessment categories (locomotor, manual activity, language, emotional status, intelligence, and social behavior) and consists of 24 items in each of the six categories. As noted, for the purposes of this study, we used only the locomotor assessment. Example items and passing rates were "The child walks well" ( $81 \%$ or more at $2-3$ months to one year) and "Jumps several steps with one foot" ( $81 \%$ or more at $8-11$ months to 3 years). Parents were asked to designate whether the child was "capable of" or "incapable of" each motor ability item. Success on each item was scored plus $(+)$ and failures were scored minus $(-)$. If the child was able to perform three items successively, we assumed that he/she could perform all items at a lower level. Similarly, if the child failed three items successively, it was assumed that he/she would fail all items of a higher level. When the child was not able to continuously do more than three items, performance level was determined after answering "capable" in two cases. For example, one child was able to perform item number 10 but was not able to perform item number 9 and item number 11 . We determined performance level at the age that added one pass to the consecutive passes.

2.3. Data Analysis. Each of the 67 items of the AHEMD-SR was arranged to match 1 of the 20 variables, which were classified into five subscales according to quartile values following the methods described by Rodrigues and his collaborators 
TABLE 1: Family characteristics.

\begin{tabular}{|c|c|c|c|}
\hline Family characteristics & Level & Numbers & $\%$ \\
\hline \multirow{3}{*}{ Attended children } & Less than 6 months & 3 & 1.3 \\
\hline & 6 to 12 months & 22 & 9.3 \\
\hline & More than 12 months & 212 & 89.5 \\
\hline \multirow{5}{*}{$\begin{array}{l}\text { Number of children } \\
\text { in family }\end{array}$} & 1 & 86 & 33.2 \\
\hline & 2 & 94 & 36.3 \\
\hline & 3 & 67 & 25.9 \\
\hline & 4 & 10 & 3.9 \\
\hline & 5 or more & 2 & 0.8 \\
\hline \multirow{5}{*}{$\begin{array}{l}\text { Number of rooms } \\
\text { in home }\end{array}$} & 1 & 6 & 2.3 \\
\hline & 2 & 13 & 5.0 \\
\hline & 3 & 86 & 33.0 \\
\hline & 4 & 79 & 30.3 \\
\hline & 5 or more & 77 & 29.5 \\
\hline \multirow{2}{*}{ Outside space } & Ample space & 156 & 59.5 \\
\hline & None & 106 & 40.5 \\
\hline \multirow{5}{*}{ Parents' physical fitness experience } & None & 39 & 16.5 \\
\hline & Less than 1 year & 13 & 5.5 \\
\hline & From 1 to under 2 years & 17 & 7.2 \\
\hline & From 2 to under 3 years & 38 & 16.0 \\
\hline & More than 3 years & 130 & 54.9 \\
\hline \multirow{5}{*}{ Parents' level of physical activity } & Very good & 38 & 16 \\
\hline & A little good & 80 & 33.6 \\
\hline & Neither good nor bad & 71 & 29.8 \\
\hline & A little weak & 30 & 12.6 \\
\hline & Very weak & 19 & 8.0 \\
\hline
\end{tabular}

[8]. In addition, a total AHEMD-SR score was calculated by adding the scores for each subscale.

For motor ability (Enjoji Infant Analytic Developmental Test), estimates of degree of association between parental physical activity experience and number of siblings were determined using Student's $t$-tests and analysis of variance (ANOVA) procedures.

\section{Results}

3.1. Family Characteristics. Table 1 provides information for family characteristics. In this investigation, most children had taken part in childcare for 12 months or more. Most families had 1-3 children, and the majority of families had more than 3 rooms in their home. Nearly $60 \%$ of parents felt that they had enough outdoor space for their children's physical activities. About $50 \%$ had physical fitness experience of 3 years or more, and more than $30 \%$ felt that their activity level was good.

3.2. Reliability of the Japanese Versions of the AHEMD-SR. Cronbach's $\alpha$ was used to test internal consistency for the 20 variables; a value of $\alpha=.78$ confirmed reliability. A similar finding was noted for the five subscales and total score: $\alpha=.71$. Pearson's correlations between total AHEMDSR score and each of the five subscale scores revealed significant values $(P<.01)$ in a positive direction ranging from .28 to .78; the highest values were with FMT (.78) and GMT (.72). These results confirm the reliability of the Japanese version of the AHEMD-SR and suggest that Fine and Gross Motor Toys had the strongest influence on children's level of motor ability.

3.3. Validity of the Japanese Version of the AHEMD-SR. Measurement validity was examined by investigating the relationship between developmental level of locomotor movement and AHEMD-SR results. For locomotor movement, items that exist within the development age range of 18-42 months, $60 \%$ or more at the passage rate, and parents self-reported on those items by the method of the alternative ("capable of" or "incapable of"), were selected. Those values were analyzed using the method described with in the Enjoji test.

The developmental level of motor ability was in the range of 13-47 months ( $\mathrm{M}=37.84$ months, $\mathrm{SD}=9.2$ months). The relationship between motor developmental age and chronological age was used to classify children into three groups: high (motor age was higher than chronological age; 196 subjects), average (motor age and chronological age were the same; 6 subjects), and low (motor age was 
TABLE 2: Correlations between AHEMD-SR score and motor ability.

\begin{tabular}{lcc}
\hline Subscales & High/average & Low \\
\hline Outside space (OS) & $3.0(0.77)$ & $3.0(0.74)$ \\
Inside space (IS) & $2.5(1.12)$ & $2.3(1.18)$ \\
Variety of stimulation (VS) & $3.5(0.87)$ & $3.6(0.77)$ \\
Fine Motor Toys (FMT) & $2.3(0.92)$ & $1.8(0.68)^{*}$ \\
Gross Motor Toys (GMT) & $1.9(0.89)$ & $1.4(0.65)^{*}$ \\
Total AHEMD-SR & $14.7(2.19)$ & $13.8(1.48)^{+}$ \\
\hline
\end{tabular}

${ }^{*} P<0.05,{ }^{+} 0.1>P>0.05$.

lower than chronological age; 34 subjects). Given that there were only six subjects in the average group, the average and high groups were combined into a high/average group containing children whose motor age matched or exceeded their chronological age. Student's $t$-tests were performed to compare the high/average group and the low group for each of the five subscale scores and the total score of the AHEMD-SR (Table 2). Test results revealed significant differences in FMT and GMT $(P<.005)$ with the high/average group scoring higher than the Low group on both subscales. For AHEMDSR total score; a similar although not significant tendency was found $(P=.067)$, suggesting that the total score was related to the child's level of motor ability.

\subsection{Estimations of Degree of Association between Parental Physical Activity (PA) and the AHEMD-SR}

3.4.1. Influence of Parents' Physical Experience on AHEMD$S R$. Continuous PA participation from elementary school to the present was determined for parents (Table 1). Parents without any habitual (regular) PA experience numbered 39, while 198 parents indicated some level of PA experience (less than 1 year: 13 ; $1-2$ years: 17 ; $2-3$ years: 38 ; and 3 years or more: 130). To determine whether the parents' level of PA experience (none or some) was correlated with AHEMD-SR scores, Student's $t$-tests were performed between experience groups. Table 3 shows that a significant difference was found for GMT $(t=-2.128, d f=229$, and $P<.05)$, and overall, AHEMD-SR scores were higher for children of parents who had some level of PA experience than for children whose parents indicated no PA experience. These results suggest that the parents' PA experience influenced the selection of play equipment.

We also investigated whether the parents' judgment of their own activity level as "good" or "weak" influenced the composition of the home environment in regard to promoting the child's motor development. Parents were divided into three groups based on activity level: good (including both very good and a little good). Neither good nor bad, and weak (including both very weak and a little weak). Those values and AHEMD-SR scores were subjected to a one-way analysis of variance. The effect of the parents' self-reported activity level was significant for FMT, GMT, and total AHEMD-SR $($ FMT: $F(2,225)=4.602, P<.05$; GMT: $F(2,229)=3.366, P<$ .05 ; total AHEMD-SR: $F(2,130)=4.219, P<.05)$. Post hoc analysis using the Tukey test was performed for FMT, GMT, and total AHEMD-SR. Significant differences were found between the good and weak groups for all three factors $(P<$ $.05)$. These results indicate that parents that recognize their PA level as good create a home environment that promotes their children's motor development compared with parents who recognize their PA level as weak.

3.4.2. Estimates of Association between Number of Siblings and AHEMD-SR. Table 3 presents the relation between the number of siblings and affordances in the home environment. The 259 participating families were divided into three groups based on number of children (1,2, and 3 or more children). Total AHEMD-SR scores for each group were subjected to one-way analysis of variance. The main effect of VS, FMT, GMT, and total AHEMD-SR score was significant (VS: $F(2,245)=7.603, P<.05$; FMT: $F(2,245)=8.588, P<.001$; GMT: $F(2,249)=5.252, P<.01$; total AHEMD-SR: $F(2,144)=$ 4.528, $P<.05)$. Post hoc analysis revealed that for the three factors (VS, FMT, and total AHEMD-SR), the scores for families with 1 child were significantly lower than for families with three or more children $(P<.05)$. For GMT, however, the scores for families with three or more children were significantly higher than scores for the other two groups $(P<$ $.05)$. These results indicate that families with three or more children have home environments sufficient for promoting motor development.

\section{Discussion}

With the present study, we translated the English version of the AHEMD-SR into Japanese and examined the relationship between Japanese children's (18-42 months of age) motor ability and affordances associated with the home environment. First of all, validity and reliability of the Japanese version of the AHEMD-SR were confirmed. Overall, our findings suggest that the amount of motor affordances had a positive influence on motor ability. This finding supports an earlier report by Sugihara and his collaborators [11] that young Japanese children's motor ability (ages 4-6 years) was significantly influenced by the amount of play equipment in the home. As noted earlier, one feature of this study, not examined in similar studies using the AHEMD-SR, was parental PA experience and its association with child motor development. Our results indicated that AHEMD-SR scores were higher for children of parents who had some level of $\mathrm{PA}$ experience compared to those parents indicating no PA experience. Moreover, AHEMD-SR scores were higher for homes of parents rating their PA level as good compared to those rated as weak. Sugihara and his collaborators [11] indicated that the motor ability of young children whom parents want them to become sports players in the future was higher than those of young children whom parents did not want their children to become sports players. That finding and our observations suggest that parent's values influence children's motor experience.

Whereas a comparison between data found here and AHEMD-SR normative values was not of primary interest, AHEMD-SR total score was significantly higher for Japanese children. The same outcome was not found for FMT and 
TABLE 3: Correlations between AHEMD-SR scores and motor experience of parents' level of physical activity (PA) and number of children in the family.

\begin{tabular}{|c|c|c|c|c|c|c|c|c|}
\hline \multirow{2}{*}{ Subscales } & \multicolumn{2}{|c|}{ Motor experience of parents } & \multicolumn{3}{|c|}{ Parents' level of PA } & \multicolumn{3}{|c|}{ Number of children in the family } \\
\hline & None & Some & Good & Neither good nor bad & Weak & 1 child & 2 & 3 or more \\
\hline Outside space (OS) & $2.9(0.70)$ & $3.0(0.78)$ & $3.0(0.76)$ & $3.0(0.77)$ & $2.8(0.79)$ & $2.9(0.82)$ & $3.1(0.68)$ & $2.9(0.77)$ \\
\hline Inside space (IS) & $2.3(1.14)$ & $2.45(1.13)$ & $2.6(1.18)$ & $2.5(1.11)$ & $2.2(1.05)$ & $2.4(1.13)$ & $2.4(1.17)$ & $2.7(1.08)$ \\
\hline Variety of stimulation (VS) & $3.3(1.06)$ & $3.6(0.81)$ & $3.5(0.90)$ & $3.6(0.81)$ & $3.5(0.85)$ & $3.3(1.02)$ & $3.5(0.87)$ & $3.8(0.64)$ \\
\hline Fine Motor Toys (FMT) & $1.9(0.87)$ & $2.2(0.90)$ & $2.3(0.94)$ & $2.3(0.88)$ & $1.8(0.75)$ & $2.0(0.87)$ & $2.2(0.84)$ & $2.5(0.94)$ \\
\hline Gross Motor Toys (GMT) & $1.5(0.83)$ & $1.9(0.88)$ & $1.9(0.92)$ & $1.9(0.91)$ & $1.5(0.65)$ & $1.7(0.84)$ & $1.7(0.85)$ & $2.1(0.97)$ \\
\hline Total AHEMD-SR & $13.7(1.46)$ & $14.6(2.21)$ & $14.9(2.35)$ & 14.5 (1.99) & $13.5(1.32)$ & $14.0(2.41)$ & $14.5(1.80)$ & $15.3(2.25)$ \\
\hline
\end{tabular}

GMT with values being lower. Interestingly, for our sample those two subscales had the strongest influence on status of motor ability. That overall observation very likely reflects cross-cultural differences in home affordances and child motor development.

We also feel the need to point some limitations of this work. First of all, the motor assessment used here, the Enjoji Infant Analytic Developmental Test, has seen limited use outside of Japan. Whereas we feel comfortable with its use with Japanese children, any future comparative studies may be problematic. Second, the Enjoji test scores infants' motor ability via parental subjective report, a situation that may have influenced a more positive bias reflected in the high number of participants scoring in the high category. In reference to the reliability data, we acknowledge that some of the subscales from AHEMD-SR are not unidimensional suggesting that the alpha is likely a lower bound estimate of the subscale's true reliability. Whereas the overall results suggest that the subscales and total scale are reliable, future inquiry should include other measures such as test-retest.

The current study adds to an increasing body of evidence indicating that affordances in the home environment that promote fine and gross motor skills influence child motor development. Furthermore, our results show that parental views about exercise, sports, and number of siblings in the home can have a positive impact on children motor development. Future research might seek to clarify affordances in the home environment for motor development by looking more closely at gender distinctions and culturespecific characteristics.

\section{References}

[1] S. Son and F. J. Morrison, "The nature and impact of changes in home learning environment on development of language and academic skills in preschool children," Developmental Psychology, vol. 46, no. 5, pp. 1103-1118, 2010.

[2] R. H. Bradley, B. M. Caldwell, S. L. Rock et al., "Home environment and cognitive development in the first 3 years of life: a collaborative study involving six sites and three ethnic groups in North America," Developmental Psychology, vol. 25, no. 2, pp. 217-235, 1989.

[3] D. Mundfrom, R. Bradley, and L. Whiteside, "A factor analytic study of the infant-toddler and early childhood versions of the HOME inventory," Educational and Psychological Measurement, vol. 53, pp. 479-489, 1993.
[4] A. L. Abbott, D. J. Bartlett, J. E. K. Fanning, and J. Kramer, "Infant motor development and aspects of the home environment," Pediatric Physical Therapy, vol. 12, no. 2, pp. 62-67, 2000.

[5] J. J. Gibson, The Ecological Approach to Visual Perception, Houghton Mifflin, Boston, Mass, USA, 1979.

[6] T. A. Stoffregen, "Affordances and events," Ecological Psychology, vol. 12, no. 1, pp. 1-28, 2000.

[7] N. Hirose, "An ecological approach to embodiment and cognition," Cognitive Systems Research, vol. 3, no. 3, pp. 289-299, 2002.

[8] L. P. Rodrigues, L. Saraiva, and C. Gabbard, "Development and construct validation of an inventory for assessing the home environment for motor development," Research Quarterly for Exercise and Sport, vol. 76, no. 2, pp. 140-148, 2005.

[9] P. Caçola, C. Gabbard, D. C. C. Santos, and A. C. T. Batistela, "Development of the affordances in the home environment for motor development-Infant scale," Pediatrics International, vol. 53 , no. 6, pp. 820-825, 2011.

[10] C. Gabbard, P. Caçola, and L. P. Rodrigues, "A new inventory for assessing affordances in the home environment for motor development (AHEMD-SR)," Early Childhood Education Journal, vol. 36, no. 1, pp. 5-9, 2008.

[11] T. Sugihara, S. Mori, and I. Yoshida, "Change over time in preschool children's motor ability and structural analysis of environmental factors," Research Report of Ministry of Education, Culture, Sports, Science and Technology Grants-in-Aid for Scientific Research B in 2002-2003, 2004, (Japanese).

[12] A. Haydari, P. Askari, and M. Nezhad, "Relationship between affordances in the home environment and motor development in children age 18-24 months," Journal of Social Science, vol. 5, pp. 319-328, 2009.

[13] Y. Hsieh, A. Hwang, H. Liao, P. Chen, W. Hsieh, and P. Chu, "Psychometric properties of a Chinese version of the home environment measure for motor development," Disability and Rehabilitation, vol. 33, no. 25-26, pp. 2454-2463, 2011.

[14] A. F. Miquelote, D. C. C. Santos, P. M. Caçola, M. I. Montebelo, and C. Gabbard, "Effect of the home environment on motor and cognitive behavior of infants," Infant Behavior \& Development, vol. 35, pp. 329-334, 2012.

[15] V. A. Temple, P. Naylor, R. E. Rhodes, and J. W. Higgins, "Physical activity of children in family child care," Applied Physiology, Nutrition and Metabolism, vol. 34, no. 4, pp. 794-798, 2009.

[16] M. Enjoji and N. Yanai, "Analytic test for development in infancy and childhood," Japanese Pediatric Society, vol. 4, no. 7, pp. 2-6, 1961.

[17] M. Enjoji, Infant Analytic Developmental Test of the EnjojiNew Revised Edition Type-Pediatrics Department in Kyushu University, Keio University, Tokyo, Japan, 2009, (Japanese). 

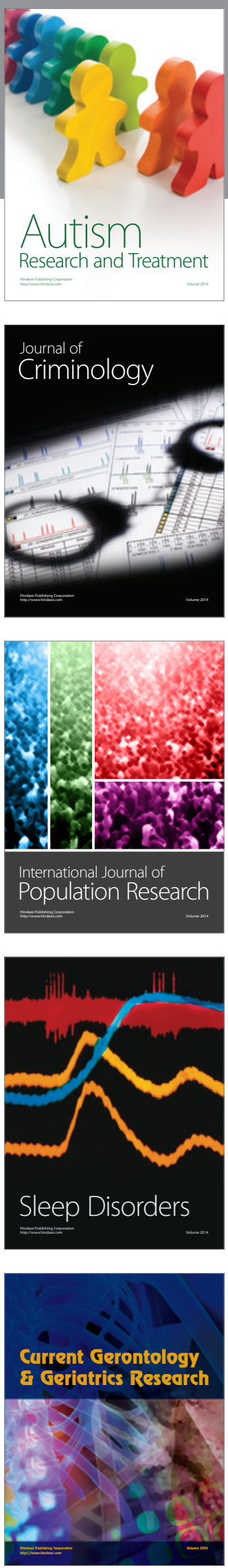
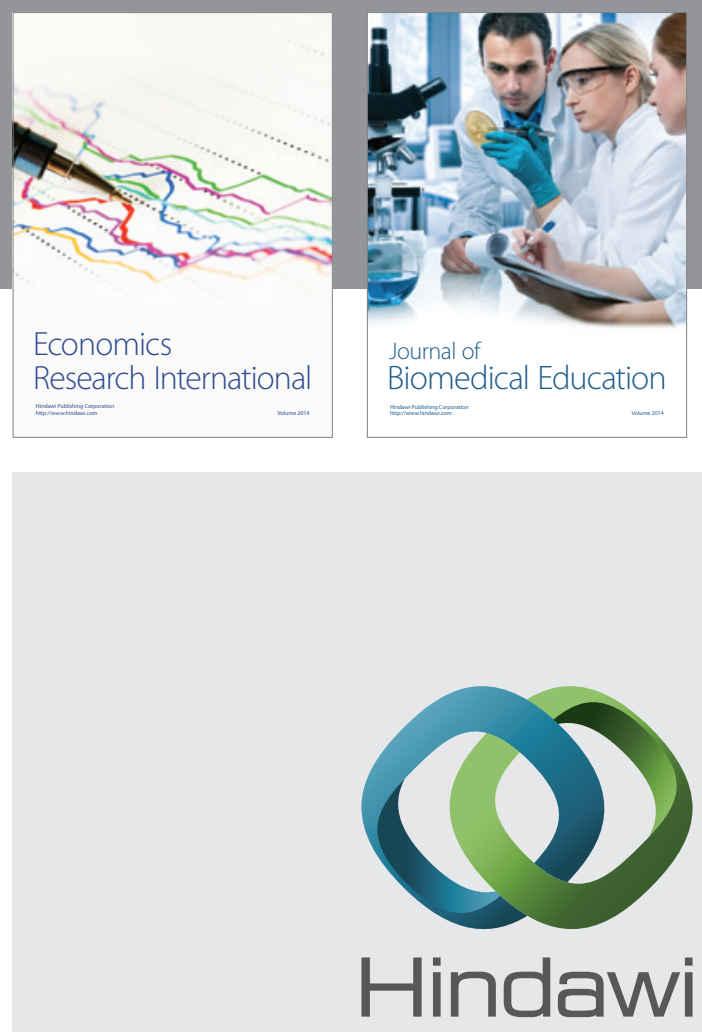

Submit your manuscripts at

http://www.hindawi.com
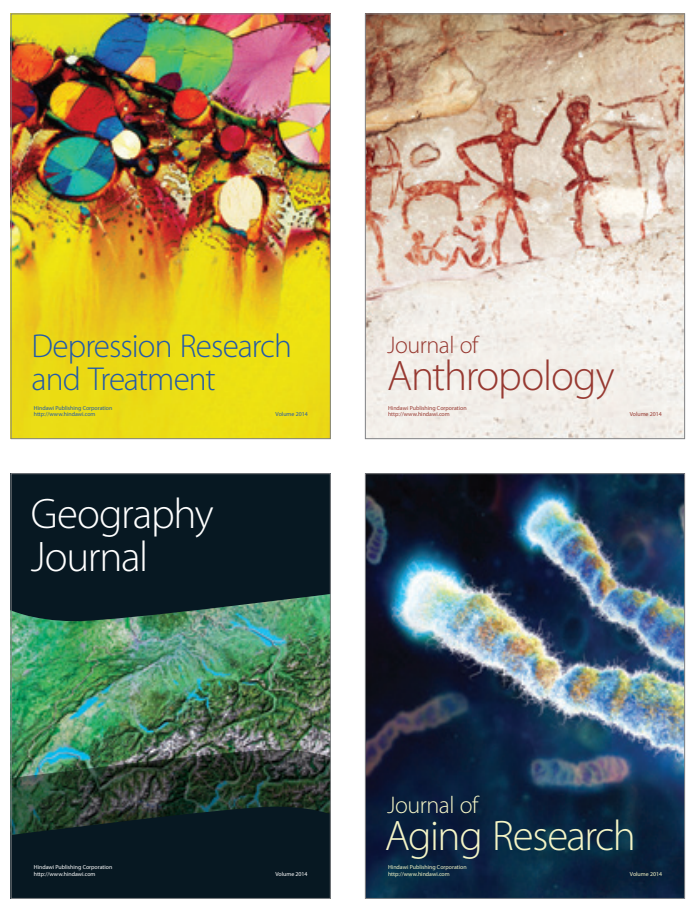
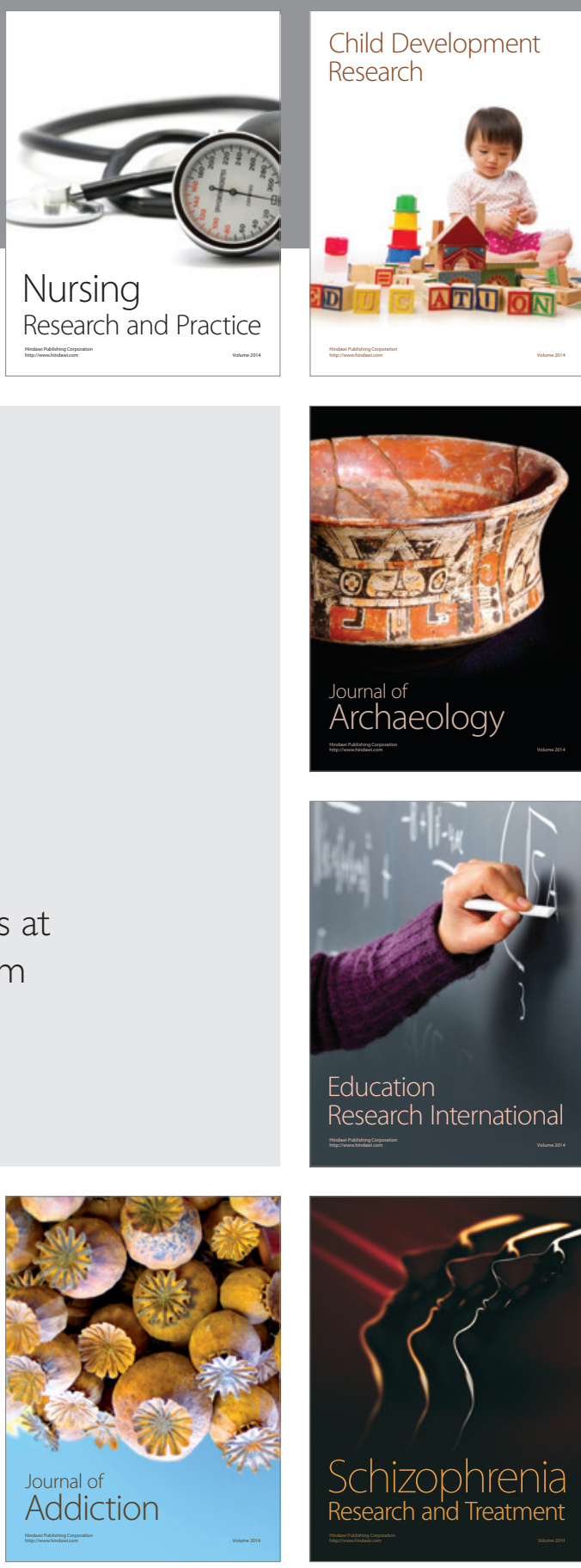

(D)
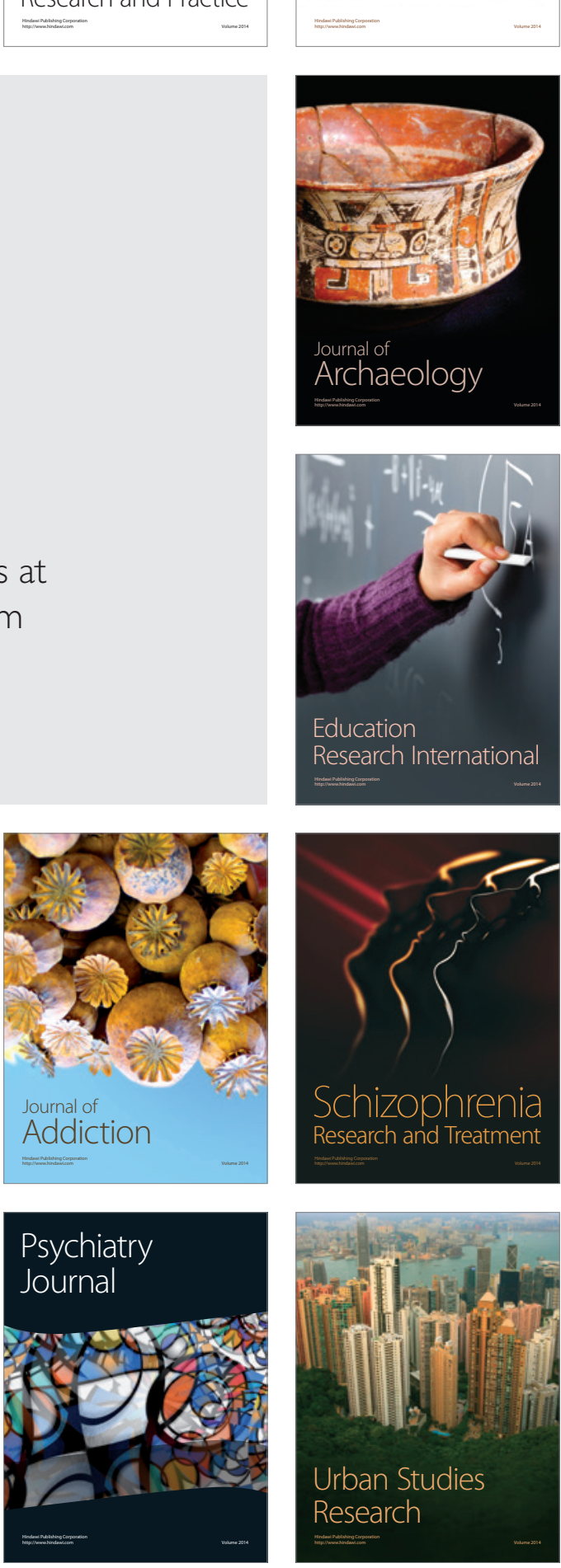